\title{
Microbial Purification of Postfermentation Medium after 1,3-PD Production from Raw Glycerol
}

\author{
Daria Szymanowska-Powałowska, Joanna Piątkowska, and Katarzyna Leja \\ Department of Biotechnology and Food Microbiology, Poznań University of Life Sciences, ul. Wojska Polskiego 48, \\ 60-527 Poznan, Poland \\ Correspondence should be addressed to Daria Szymanowska-Powałowska; darszy@up.poznan.pl
}

Received 15 July 2013; Revised 28 August 2013; Accepted 30 August 2013

Academic Editor: Kannan Pakshirajan

Copyright (C) 2013 Daria Szymanowska-Powałowska et al. This is an open access article distributed under the Creative Commons Attribution License, which permits unrestricted use, distribution, and reproduction in any medium, provided the original work is properly cited.

\begin{abstract}
1,3-Propanediol (1,3-PD) is an important chemical product which can be used to produce polyesters, polyether, and polyurethanes. In the process of conversion of glycerol to 1,3-PD by Clostridium large number of byproducts (butyric, acetic and lactic acid) are generated in the fermentation medium. The aim of this work was to isolate bacteria strains capable of the utilization of these byproducts. Screening of 30 bacterial strains was performed using organic acids as carbon source. Selected isolates were taxonomically characterized and identified as Alcaligenes faecalis and Bacillus licheniformis. The most active strains, Alcaligenes faecalis JP1 and Bacillus licheniformis JP19, were able to utilize organic acids almost totally. Finally, it was find out that by the use of coculture (C. butyricum DSP1 and A. faecalis JP1) increased volumetric productivity of 1,3-PD production (1.07 g/L/h) and the yield equal to $0.53 \mathrm{~g} / \mathrm{g}$ were obtained in bioreactor fermentation. Moreover, the only by-product present was butyric acid in a concentration below $1 \mathrm{~g} / \mathrm{L}$.
\end{abstract}

\section{Introduction}

As the production of biofuels from raw materials continuously increases, optimization of production processes is necessary. A very important issue is the development of wasteless methods of biodiesel production. One way of utilization of glycerol generated in biodiesel production is its microbial conversion to 1,3-PD [1]. However, during this process different accompanying metabolites, such as organic acids and ethanol, are also synthesized from which the main product, 1,3-PD, must be separated [2,3]. Difficulties in its separation arise from its high boiling point and the presence of two hydroxyl groups which make it strongly hydrophilic, and therefore complicate its extraction. There are many methods of purification of 1,3-PD $[4,5]$; however, physical and mechanical methods of purification are very expensive. An effective method of 1,3-PD separation is an evaporation process coupled to vacuum distillation. However, the main disadvantages of that method are high demand for energy, and, moreover, the need to remove proteins and salts before this process. Other methods of purification also have limitations. Electrodialysis, used for desalination before evaporation, causes low product yield due to losses. Liquid-liquid extraction requires large amounts of solvent. Cyclic sorption and desorption on zeolite also poses problems, such as the requirement of dewatering step and high chance of contamination (due to uninterrupted link between the bioreactor and the separation equipment). Reactive extraction is a relatively complicated process. The removal of proteins, ethanol, and salts is necessary before the reaction. Additionally, trace amounts of aldehyde in 1,3PD interferes with the polymerization of PTT. Aqueous twophase extraction requires large amounts of methanol and difficulty in separation of the two alcohols occur. Chromatography consumes more energy than the simple evaporation and distillation, and while high overall purity and yield of 1,3-PD can be obtained, the resulting 1,3-PD solution is not concentrated but diluted because of the low selectivity and capacity of resin or absorbent. Additionally, the efficiency of 
chromatographic matrices is susceptible to deterioration if feeds are not desalinated and deproteinized [4].

Alternative purification solutions are under investigation, among which microbiological ways of utilization of byproducts are very interesting and promising [6-8]. Many reports exist concerning the use of coculture in 1,3-PD production [9-11]. There is, however, a lack of information on cocultures formed by a 1,3-PD producer and a microorganism capable of utilization of 1,3-PD by-products. Such a solution could result in better overall process productivity and facilitate the downstream processing.

The aim of this work was the isolation of new strains able to utilize organic acids, as sole carbon sources, which are generated during the production of 1,3-PD from glycerol. Furthermore, the possibility of enhancing the kinetic parameters of microbial 1,3-PD productions by the use of coculture were investigated.

\section{Materials and Methods}

2.1. Pure Culture Inoculum. In the conversion process of raw glycerol to 1,3-PD a bacteria strain, C. butyricum DSP1, was used. C. butyricum DSP1 was previously isolated from ruminal fluid and collected in the Department of Biotechnology and Food Microbiology, Poznan University of Life Sciences Poland, and deposited at the Polish Collection of Microorganisms PCM.

The strain was maintained in Reinforced Clostridial Medium (RCM, Oxoid, UK) in serum bottles at $4^{\circ} \mathrm{C}$. Precultures of pure culture inoculum were cultivated in Hungate test tubes in appropriate cultivation media $\left(37^{\circ} \mathrm{C}, 18 \mathrm{~h}\right)$. Bacteria from the genus Clostridium were cultured in a chamber for cultivation of anaerobic microorganisms (the Whitley MG500, Don Whitley Scientific, Shipley, UK), without pH regulation and stirring.

The enrichment of isolated bacteria $A$. faecalis biomass was carried out in medium (CM) consisting of (per liter deionized water): $2.0 \mathrm{~g} \mathrm{C}_{2} \mathrm{H}_{7} \mathrm{NO}_{2}, 2.0 \mathrm{~g} \mathrm{~K}_{2} \mathrm{HPO}_{4}, 0.2 \mathrm{~g}$ $\mathrm{MgSO}_{4}$ and $50 \mu \mathrm{L}$ solution of $\mathrm{CaCl}_{2}$. Precultures of pure culture inoculum were incubated under relative anaerobic conditions in an incubation chamber $\left(32^{\circ} \mathrm{C}, 20 \mathrm{~h}\right)$.

2.2. Isolation and Screening Medium. The isolation medium for new strains was Nutrient Broth Agar and glucose (2\%). The composition of the screening medium was (per liter deionized water): $33.5 \mathrm{~g} \mathrm{C}_{3} \mathrm{H}_{8} \mathrm{O}_{2}, 4.65 \mathrm{~g} \mathrm{C}_{3} \mathrm{H}_{7} \mathrm{COOH}, 1.69 \mathrm{~g}$ $\mathrm{CH}_{3} \mathrm{COOH}, 2.76 \mathrm{~g} \mathrm{C}_{2} \mathrm{H}_{4} \mathrm{OHCOOH}, 3.0 \mathrm{~g} \mathrm{C}_{3} \mathrm{H}_{5}(\mathrm{OH})_{3}$, and $0.34 \mathrm{~g} \mathrm{C}_{2} \mathrm{H}_{5} \mathrm{OH}$.

2.3. Isolation Process. Bacteria strains able to organic acids utilization were isolated from typical sources of methanogenic fermentations (fermented chocolate, ensilages, and slurry). The first stage in the isolation of the desired strains was based on the pour-plate method. After the incubation period $\left(48 \mathrm{~h}, 32^{\circ} \mathrm{C}\right)$, single colonies that had different morphological traits and the characteristics of a given bacterium genus were collected using disposable loops. Cells were maintained at $-80^{\circ} \mathrm{C}$ in the culture broth supplemented with $20 \%$ glycerol.

2.4. Screening. Obtained isolates were cultivated on postfermentation broth. After the production of 1,3-PD from glycerol and biomass separation, permeate was inoculated by $A$. faecalis and $B$. licheniformis bacteria isolates $(10 \% \mathrm{v} / \mathrm{v})$. The process was carried out for 7 days, in $32^{\circ} \mathrm{C}$, without $\mathrm{pH}$ regulation $(\mathrm{pH}$ at the beginning of the process adjusted to 7.15 ), and in strictly anaerobic conditions. Samples were taken in 24 hours intervals - the changes of glycerol, 1,3-PD, and organic acids were analyzed by HPLC.

2.5. Bacterial Identification. Total DNA from bacteria was extracted with Genomic Mini AX Bacteria Kit (A \& A Biotechnology, Gdansk, Poland) after an initial $1 \mathrm{~h}$ incubation in $50.0 \mathrm{mg} / \mathrm{mL}$ lysozyme (Sigma, Poland) at $37^{\circ} \mathrm{C}$. Sequences encoding small subunits of $16 \mathrm{~S}$ rRNA were amplified by PCR using SDBact0008aS20 and SUniv1492bA21 primers [12]. The PCR products were purified using the Clean-up Kit (A \& A Biotechnology, Gdansk, Poland) and sequenced at Genomed (Warsaw, Poland) using the primers used for PCR and a primer for an inner sequence (GTGCCAGCMGCCGCCCTAA). The sequences obtained were arranged into contigs and identified using the BLAST service of the GenBank database [13].

2.6. Phylogenetic Analyses. The sequences encoding the $16 \mathrm{~S}$ rRNA of $C$. butyricum DSP1 and A. faecalis strains were compared with the randomly selected sequences of Clostridium sp. and Alcaligenes sp. in GenBank. The sequences were aligned using the ClustalW program as implemented in BioEdit (version 7.0.9). The phylogenetic analyses were conducted using the MEGA 4.0 software [12]. The neighbourjoining method was used for phylogenetic reconstruction, and the p-distance was used for distance analysis. The best phylogenetic distance tree is shown.

2.7. Fermentation Medium. The composition of the fermentation medium was (per liter deionized water): $0.26 \mathrm{~g} \mathrm{~K}_{2} \mathrm{HPO}_{4}$; $0.02 \mathrm{~g} \mathrm{KH}_{2} \mathrm{PO}_{4} ; 1.23 \mathrm{~g}\left(\mathrm{NH}_{4}\right)_{2} \mathrm{SO}_{4} ; 0.1 \mathrm{~g} \mathrm{MgSO}_{4} \times 7 \mathrm{H}_{2} \mathrm{O}$; $0.01 \mathrm{~g} \mathrm{CaCl}_{2} \times 2 \mathrm{H}_{2} \mathrm{O} ; 0.01 \mathrm{~g} \mathrm{FeCl} 2 \times 7 \mathrm{H}_{2} \mathrm{O}$, and $2.0 \mathrm{~g}$ yeast extract. The fermentation medium was supplemented with crude glycerol (Wratislavia-Bio, Wroclaw, Poland) at a concentration of $80.0 \pm 1.0 \mathrm{~g} / \mathrm{L}$ in batch fermentation. The crude glycerol composition was (w/w) 85.6\% glycerol, 6\% $\mathrm{NaCl}, 11.2 \%$ moisture, and $\mathrm{pH}$ 6.5. The media were autoclaved (121 ${ }^{\circ} \mathrm{C}, 20 \mathrm{~min}$.).

2.8. Fermentation Experiments. Fermentations were carried out in bioreactor (2L) (Sartorius Stedim, Germany). The temperature of the process was $37^{\circ} \mathrm{C}$, stirring rate was $60 \mathrm{rpm}$, $\mathrm{pH}$ was automatically regulated with $5 \mathrm{M} \mathrm{NaOH}$ at $7.0 \pm 0.1$. The process was carried out using two bacteria strains. At the beginning of the process, bacteria were grown separately in two vessels $(200 \mathrm{~mL})$ connected to the bioreactor. In the first vessel C. butyricum DSP1 was cultivated on RCM medium, and in the second, A. faecalis JP1 was cultivated on CM 
medium. This preliminary stage was continued for 18-20 hours. After this time, the medium in the bioreactor was inoculated with C. butyricum DSP1 (using peristaltic pump) and the synthesis of 1,3-PD started. When the concentration of metabolites (both 1,3-PD and organic acids) increased, the second bacteria culture-A. feacalis JP1-was fed into the bioreactor. Samples from the bioreactor were taken at intervals every 2-5 hours. All data presented are means of two independent experiments performed under the same culture conditions.

2.9. Analytical Methods. 1,3-PD, glycerol, and organic acids were assayed by high performance liquid chromatography.

Samples for chemical analysis were first centrifuged at $10,000 \mathrm{~g}$ for $10 \mathrm{~min}$ at $4^{\circ} \mathrm{C}$ (Multifuge 3SR, Germany), filtered through a $0.22 \mu \mathrm{m}$ membrane filter (Millex-GS, Millipore, USA), and then analyzed on an HPLC system (Agilent Technologies 1200 series).

Agilent Technolgies 1200 series system equipped with a refractive index detector was used. Analyses were performed isocratically at a flow rate of $0.6 \mathrm{~mL} / \mathrm{min}$. on an Aminex HPX-87H $300 \times 7.8$ column (Bio-Rad, CA, USA) at a constant temperature of $65^{\circ} \mathrm{C} . \mathrm{H}_{2} \mathrm{SO}_{4}(0.5 \mathrm{mN})$ was the mobile phase. External standards were applied for identification and quantification of peaks area. Retention times (Rt) determined for the targeted compounds for were as follows: 1,3-PD-17.17 $\mathrm{min}$; glycerol-13.03 min; butyric acid-20.57 min; acetic acid-14.4 min; lactic acid$11.19 \mathrm{~min}$; ethanol-21.34 $\mathrm{min}$.

2.9.1. Determination of Interactions between of A. feacalis Jp1, B. licheniformis Jp19 and C. butyricum Dsp1. In order to verify if any antagonisms exist between the tested $A$. feacalis JP1 and B. licheniformis JP19 strains in relation to C. butyricum DSP1 analyses were conducted, including preparation of culture media for A. feacalis JP1 and B. licheniformis JP19 strains, separation of culture media into fractions (the supernatant and precipitate), preparation of C. butyricum DSP1 and analyses of activity of the obtained liquid culture and the supernatant by the well method.

2.9.2. Preparation of Culture Liquid Media. Antibacterial activity was determined using $24 \mathrm{~h}$ cultures of $A$. feacalis JP1 (media CA) and B. licheniformis JP19 (media with 2\% glucose). Cultures were run at a temperature of $32^{\circ} \mathrm{C}$.

\subsubsection{Separation of the Culture Liquid Media into Fractions.} In order to obtain supernatants (S), the cultures of analyzed strains were centrifuged $(5000 \times \mathrm{g} ; 10 \mathrm{~min})$. Analyses of the supernatant were aimed at the determination of antagonistic activity of bacterial exocellular metabolites.

2.9.4. Preparation of Indicator Microorganisms. Indicator microorganism (C. butyricum DSP1) was transferred to test tubes containing $10 \mathrm{~mL}$ RCM medium (to proliferate biomass). Cultures were run at $37^{\circ} \mathrm{C}$ for $24 \mathrm{~h}$. Then, in order to obtain a distinct confluent layer, the liquefied agar medium was inoculated with $10 \%(\mathrm{v} / \mathrm{v}) 24 \mathrm{~h}$ culture of the indicator culture and poured onto Petri dishes.

2.9.5. Analyses of Antibacterial Activity of Liquid Culture Medium and the Supernatant Fraction. After solidification of the Tryptose Sulfite Cycloserine Agar (TSC, Oxoid, UK) inoculated with indicator microorganism, wells were made using a cork borer. Each well was supplemented with $150 \mu \mathrm{L}$ liquid culture medium or $150 \mu \mathrm{L}$ supernatant fraction of the analyzed strain. After incubation $\left(24 \mathrm{~h}, 32^{\circ} \mathrm{C}\right.$, anaerobic), the diameters of the zones of growth inhibition were measured. Bacteriostatic properties were determined by measuring the growth inhibition zone diameter (growth limitation of the indicator strain).

\section{Results}

3.1. Isolation and Characterization of Microorganisms Able to Organic Acids Utilizations. There is only little literature data about purification of postfermentation broths in the microbial 1,3-PD synthesis [10]. Chemical purification processes are expensive [4]. Thus, in this work, the perspectives of utilization of microorganisms to remove the by-products, synthesized during the production of 1,3-PD from glycerol was considered. As an isolation source probes from methanogenic fermentation were chosen. Finally, 30 bacteria isolates were obtained. These were cultured on postfermentation broth of typical composition for propanediol fermentation carried out by $C$. butyricum. The aim was to screen for microorganisms able to decrease the concentrations of organic acids and ethanol without changing the amount of 1,3-PD. Changes in concentrations of compounds in the postfermentation broth after 7 days of cultivation are presented in Table 1 .

Fifteen strains exerting the most significant influence on the broth composition (Table 1) were selected for further study. Unfortunately, some strains utilized both-organic acids and 1,3-PD. It disqualified these strains for the purpose of postfermentation broth purification. Also strains utilizing glycerol were omitted.

Nine of the obtained strains had significant influence of the amount of organic acids and had no influence on 1,3-PD level. Strains number 1, 12, 15, and 19 were able to partially or completely utilize organic acids. The best isolates were selected for identification. Five of them belonged to the Alcaligenes faecalis species and four to Bacillus licheniformis (Table 2). Some strains (of the 15 selected isolates) were able to utilize organic acids very slowly. For example, strain no 1 metabolized it in 24 hours, while strain no 15 in 120 hours. For this reason, A. faecalis JP1 (isolate no 1) was chosen for further experiments.

To determine the relationship between the C. butyricum DSP1 strain and the $A$. faecalis strains, a phylogenetic tree was built based on the nucleotide sequences of the genes encoding 16S rRNA (Figure 1).

The antibacterial activity test indicated that strain $A$. faecalis JP1 has no antagonistic activity towards $C$. butyricum 
TABLE 1: Compounds of postfermentative broth after 7 days of cultivation of the isolates.

\begin{tabular}{|c|c|c|c|c|c|c|c|}
\hline \multirow{2}{*}{ Strain number } & \multicolumn{6}{|c|}{ Utilization level } & \multirow{2}{*}{ Time } \\
\hline & Butyric acid & Acetic acid & Lactic acid & Ethanol & 1,3-PD & Glycerol & \\
\hline 1 & ++ & +++ & +++ & - & - & - & $24 \mathrm{~h}$ \\
\hline 2 & + & +++ & + & - & - & + & NA \\
\hline 3 & + & +++ & + & - & - & + & NA \\
\hline 5 & ++ & +++ & + & - & - & - & NA \\
\hline 6 & + & + & - & - & + & + & NA \\
\hline 7 & - & + & - & - & - & + & NA \\
\hline 9 & + & +++ & ++ & - & - & + & NA \\
\hline 11 & + & +++ & + & - & - & - & NA \\
\hline 12 & ++ & +++ & ++ & - & - & - & $96 \mathrm{~h}$ \\
\hline 15 & ++ & +++ & +++ & - & - & - & $120 \mathrm{~h}$ \\
\hline 17 & - & +++ & + & - & - & - & NA \\
\hline 19 & + & +++ & +++ & - & - & - & $96 \mathrm{~h}$ \\
\hline 21 & + & & ++ & - & - & - & NA \\
\hline 25 & + & - & + & - & + & + & NA \\
\hline 29 & - & - & ++ & - & + & + & NA \\
\hline
\end{tabular}

+++ : complete utilization.

++ : utilization of more than $50 \%$.

+ : insignificant utilization $(<10 \%)$.

-: lack of utilization.

${ }^{*}$ Time until $90 \%$ organic acids utilization.

NA: not applicable.

TABLE 2: Identification on new isolates by amplification of $16 \mathrm{~S}$ rRNA.

\begin{tabular}{lccc}
\hline Strains number & Isolation source & Species & Homology to geneare \\
\hline 1 & Fermented silage with manure & Alcaligenes faecalis & $99 \%$ \\
5 & Fermented silage with manure & Alcaligenes faecalis & $98 \%$ \\
12 & Fermented silage with manure & Alcaligenes faecalis & $97 \%$ \\
15 & Fermented silage with manure & Alcaligenes faecalis & $98 \%$ \\
21 & Fermented silage with manure & Alcaligenes faecalis & $96 \%$ \\
3 & Fermented chocolate & Bacillus licheniformis & $98 \%$ \\
9 & Fermented silage with manure & Bacillus licheniformis & $97 \%$ \\
11 & Fermented silage with manure & Bacillus licheniformis & $96 \%$ \\
12 & Fermented chocolate & Bacillus licheniformis & $97 \%$ \\
\hline
\end{tabular}

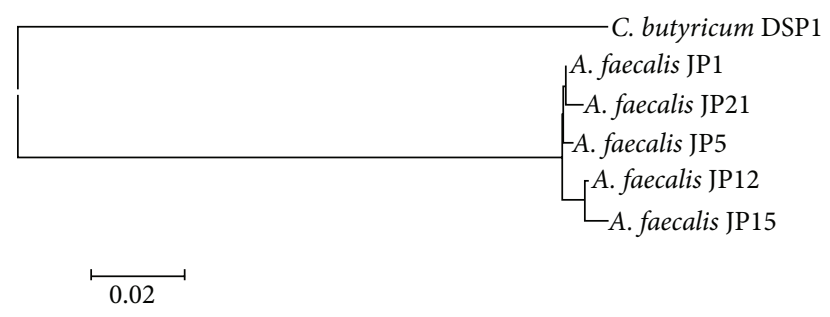

FIGURE 1: 16S rRNA-based phylogenetic tree showing the position $C$. butyricum DSP1 among related $A$. faecalis strains.

DSP1 used in 1,3-PD production. However, strains B. licheniformis inhibits growth of $C$. butyricum strain (data not shown).

3.2. Cultivation of Coculture Consisting of C. butyricum Dsp1 and A. faecalis Jp1. Fermentation by-products cause toxic stress which can damage bacteria cells, presence of these products also inhibits polymerization reactions in polyurethane production $[14,15]$. Thus, efforts were taken to remove the by-products of the glycerol conversion to 1,3-PD in a microbiological way. Two bacteria strains were used C. butyricum DSP1 and $A$. faecalis JP1. In the first step of the experiment the fermentation medium was inoculated with $C$. butyricum. The synthesis of 1,3-PD started ca. 13 hour after inoculation. Glycerol was utilized and 1,3-PD was synthesized. During the fermentation, as the amount of 1,3-PD reached $25 \mathrm{~g} / \mathrm{L}$, butyric acid level was at $1.68 \mathrm{~g} / \mathrm{L}$, lactic acid $-1.34 \mathrm{~g} / \mathrm{L}$, and acetic acid $-1.21 \mathrm{~g} / \mathrm{L}$, an inoculum culture of $A$. faecalis was added to the fermentation tank. During the first $3 \mathrm{~h}$ after inoculation with the second strain, the level of 1,3-PD was still increasing. In $32 \mathrm{~h}$ of fermentation, a significant decrease (up to 20\%) of lactic and acetic acid concentration was observed. Simultaneously, the productivity of 1,3-PD increased (between 31 and 35 


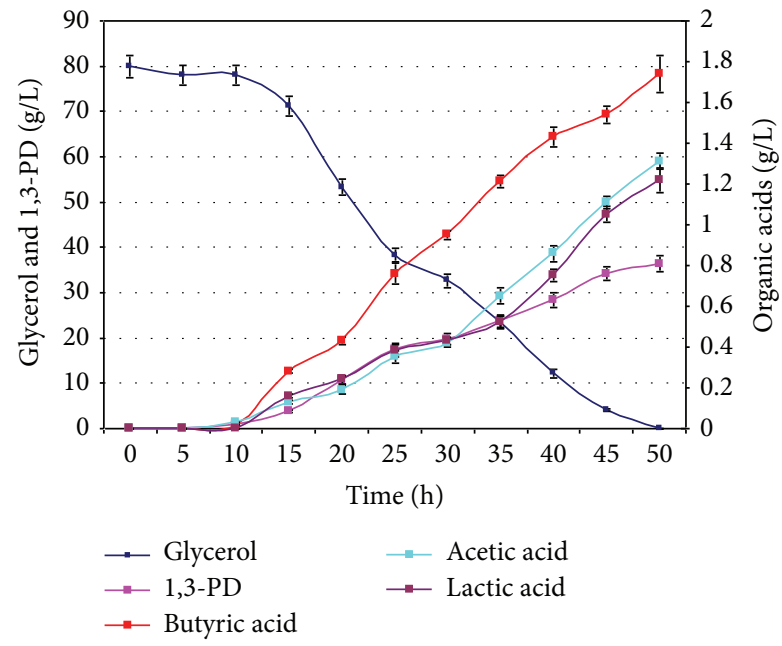

(a)

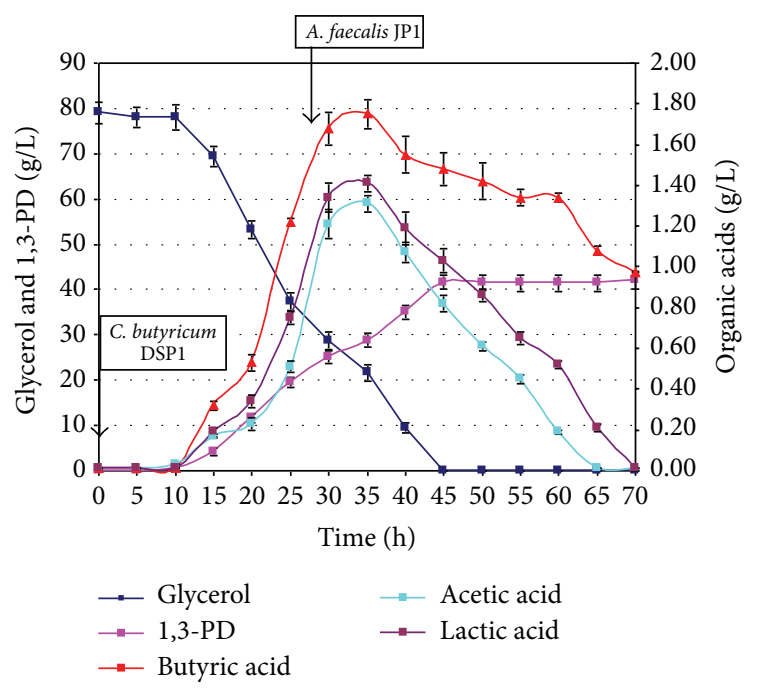

(b)

Figure 2: Changes in substrate and metabolites concentration during the conversion of glycerol to 1,3-PD by (a) monoculture of C. butyricum DSP1 and (b) coculture of C. butyricum DSP1 and A. faecalis JP1.

hour of fermentation it was equal $1.55 \mathrm{~g} / \mathrm{L} / \mathrm{h})$. Complete glycerol utilization was observed in $39 \mathrm{~h}$, the yield of 1,3-PD production was $0.53 \mathrm{~g} / \mathrm{g}$ crude glycerol. The process was carried until complete lactic and acetic acids utilization (Figure 2(b)).

\section{Discussion}

Methanogenic fermentation is a very interesting source of microorganism which can be applied in industrial processes of glycerol utilization $[10,11,16]$. The reason for this situation is probably the fact that glycerol is often added into methanogenic fermentation as an additional carbon source [17]. Thus, microorganisms obtained from this source tolerate high osmotic pressures. In this work, strains from silage fermented with manure and fermented chocolate were obtained. During methane production (in the acido- and acetogenesis steps) some organic acids are produced. Next, these acids are converted to gases. Thus, the authors selected such isolation sources to obtain strains resistant to high concentration of organic acids and able to utilize it. Finally, 30 isolates were obtained and their metabolic activity was tested. The best strains were $A$. faecalis JP1 and B. licheniformis JP19. Organic acids such as acetic acid and butyric acid are formed as by-products in the fermentation of glycerol to 1,3PD. These compounds inhibit the growth of C. butyricum and deteriorate its ability to biotransform glycerol to 1,3-PD. The acids formed increase the hydrogen ion concentration in the fermentation broth, diffuse into the cell, and can lead to its death. In order to prevent it, the activity of proton pumping proteins increases resulting in higher ATP consumption. The activation of this defense mechanism leads to decreased cell metabolic activity, lower production of biomass, and metabolites. Biebl [18] described the influence of butyric and acetic acids (apart from 1,3-PD, the main primary metabolites of propanediol fermentation) on C. butyricum. The author determined the concentrations that inhibited the growth of the bacterium: butyric acid $-19 \mathrm{~g} / \mathrm{L}$ and acetic acid $-27 \mathrm{~g} / \mathrm{L}$. Thus, the limitation by organic acid concentration during 1,3PD synthesis is a very important issue, especially in fed-batch fermentations and at the end of the process. Removal of these by-products can increase the metabolic activity of bacteria producing 1,3-PD. Colin et al. [19] examined the influence of the addition of butyrate and acetate on C. butyricum CNCM 1211 strain during conversion of glycerol into 1,3PD. The addition of these metabolites in concentrations of $2.5-15.0 \mathrm{~g} / \mathrm{L}$ distinctly affected the bacterium viability and its metabolism. Significant observations were made during the research. The addition of acetate triggered an increase in biomass concentration and butyrate production, and at the same time reduced the yield of 1,3-PD, whereas the addition of butyrate resulted in increased diol synthesis, reduction in biomass, and butyrate production. The presented results enable to propose a thesis that reduction in butyrate synthesis in the cell ensures the appropriate amount of $\mathrm{NADH}$, which is vital for the synthesis of 1,3-propanediol. In the present study, partial removal of butyric acid did not influence the number of microorganisms because the biomass already reached the plateau before inoculation with A. faecalis JP1. The decreased amounts of acetic and lactic acids obtained in cocultures were accompanied by better fermentation parameters then the control. The obtained yield and volumetric productivities were $15 \%$ and $30 \%$ higher, respectively. Other reports exist that concern the use of cocultures for enhanced 1,3-PD production [8, 9]. Selembo et al. [8] performed a model experiment with C. butyricum and Methanosarcina mazei, a microorganism capable of utilization of the 1,3PD fermentation by-products. Bizukojc et al. [9] used an unidentified coculture to convert glicerol to 1,3-PD and 
hydrogen. Hereby, selected strain of the Alcaligenes genera possesses some very beneficial properties that make it fit for cocultures with $C$. butyricum. The optimal temperature for the Alcaligenes is in the range of $30-37^{\circ} \mathrm{C}$, which complies with optimal temperature for the Clostridium. Bacteria form the Alcaligenes genera shows the ability to consume oxygen and generate anaerobic conditions necessary for C. butyricum strain. It seems that both bacteria can live in syntrophy. On the other hand, because of the determined antagonism, the isolated B. licheniformis JP19 strain may be used for the purification of 1,3-PD but not in direct coculture with $C$. butyricum. The antagonistic effects result probably from the production of proteolytic and bacteriolytic enzymes (such as n-acetylmuramoyl-1-alanine amidase, EC 3.5.1.2.8) [20]. As B. licheniformis has tolerance to high concentration of organic acids and solvents it could still be utilized after a separate fermentation process for the purpose of purification [21].

\section{Conclusions}

The application of isolates from the Alcaligenes and Bacillus genera as means of purification of postfermentation broth is a promising method of removing organic acids.

However imperfect the procedures described in this work are and further investigation is necessary, such approach gives hope that the production of biodiesel may become soon a completely wasteless process. Furthermore, the removal of toxic by-products can lead to increased efficiency of the microbial synthesis of 1,3-PD and, in result, make the process more economically viable.

\section{Conflict of Interests}

The authors declare that there is no conflict of interests regarding the publication of this paper.

\section{Acknowledgments}

The paper was prepared within the framework of project PO IG 01.01.02-00-074/09 cofunded by The European Union from The European Regional Development Fund within the framework of the Innovative Economy Operational Programme 2007-2013.

\section{References}

[1] A.-P. Zeng and W. Sabra, "Microbial production of diols as platform chemicals: recent progresses," Current Opinion in Biotechnology, vol. 22, no. 6, pp. 749-757, 2011.

[2] A.-P. Zeng, "Pathway and kinetic analysis of 1,3-propanediol production from glycerol fermentation by Clostridium butyricum," Bioprocess Engineering, vol. 14, no. 4, pp. 169-175, 1996.

[3] G. Kaur, A. K. Srivastava, and S. Chand, "Advances in biotechnological production of 1,3-propanediol," Biochemical Engineering Journal, vol. 64, pp. 106-118, 2012.

[4] Z.-L. Xiu and A.-P. Zeng, "Present state and perspective of downstream processing of biologically produced 1,3propanediol and 2,3-butanediol," Applied Microbiology and Biotechnology, vol. 78, no. 6, pp. 917-926, 2008.
[5] A.-I. Qatibi, A. Bories, and J.-L. Garcia, "Sulfate reduction and anaerobic glycerol degradation by a mixed microbial culture," Current Microbiology, vol. 22, no. 1, pp. 47-52, 1991.

[6] M. F. Temudo, G. Muyzer, R. Kleerebezem, and M. C. M. van Loosdrecht, "Diversity of microbial communities in open mixed culture fermentations: impact of the $\mathrm{pH}$ and carbon source," Applied Microbiology and Biotechnology, vol. 80, no. 6, pp. 1121-1130, 2008.

[7] W. Sabra, D. Dietz, D. Tjahjasari, and A.-P. Zeng, "Biosystems analysis and engineering of microbial consortia for industrial biotechnology," Engineering in Life Sciences, vol. 10, no. 5, pp. 407-421, 2010.

[8] P. A. Selembo, J. M. Perez, W. A. Lloyd, and B. E. Logan, "Enhanced hydrogen and 1,3-propanediol production from glycerol by fermentation using mixed cultures," Biotechnology and Bioengineering, vol. 104, no. 6, pp. 1098-1106, 2009.

[9] M. Bizukojc, D. Dietz, J. Sun, and A.-P. Zeng, "Metabolic modelling of syntrophic-like growth of a 1,3-propanediol producer, Clostridium butyricum, and a methanogenic archeon, Methanosarcina mazei, under anaerobic conditions," Bioprocess and Biosystems Engineering, vol. 33, no. 4, pp. 507-523, 2010.

[10] D. Dietz and A. P. Zeng, "Efficient production of 1, 3propanediol from fermentation of crude glycerol with mixed cultures in a simple medium," Bioprocess and Biosystems Engineering, 2013.

[11] A. Suau, R. Bonnet, M. Sutren et al., "Direct analysis of genes encoding 16S rRNA from complex communities reveals many novel molecular species within the human gut," Applied and Environmental Microbiology, vol. 65, no. 11, pp. 4799-4807, 1999.

[12] K. Tamura, J. Dudley, M. Nei, and S. Kumar, "MEGA4: molecular Evolutionary Genetics Analysis (MEGA) software version 4.0," Molecular Biology and Evolution, vol. 24, no. 8, pp. 15961599, 2007.

[13] A.-P. Zeng, A. Ross, H. Biebl, C. Tag, B. Gunzel, and W. D. Deckwer, "Multiple product inhibition and growth modeling of Clostridium butyricum and Klebsiella pneumoniae in glycerol fermentation," Biotechnology and Bioengineering, vol. 44, no. 8, pp. 902-911, 1994.

[14] P. Kubiak, K. Leja, K. Myszka et al., "Physiological predisposition of various Clostridium species to synthetize 1, 3propanediol from glycerol," Process Biochemistry, vol. 47, pp. 308-1319, 2012.

[15] D. Szymanowska-Powałowska, P. Kubiak, and A. Lewicki, “The methane fermentation medium as an attractive source of bacteria from genus Clostridium capable of converting glycerol into 1, 3-propylene glycol," Journal of Biotechnology, Computational Biology and Bionanotechnology, vol. 93, no. 1, pp. 68-77, 2012.

[16] M. S. Fountoulakis and T. Manios, "Enhanced methane and hydrogen production from municipal solid waste and agroindustrial by-products co-digested with crude glycerol," Bioresource Technology, vol. 100, no. 12, pp. 3043-3047, 2009.

[17] D. R. Boone, R. W. Castenholz, G. M. Garrity, D. J. Brenner, N. R. Krieg, and J. T. Staley, Bergey's Manual of Systematic Bacteriology, The Williams \& Wilkins Company, Baltimore, Md, USA, 2005.

[18] H. Biebl, "Glycerol fermentation of 1,3-propanediol by Clostridium butyricum. Measurement of product inhibition by use of a pH-auxostat," Applied Microbiology and Biotechnology, vol. 35, no. 6, pp. 701-705, 1991.

[19] T. Colin, A. Bories, C. Lavigne, and G. Moulin, "Effects of acetate and butyrate during glycerol fermentation by Clostridium butyricum," Current Microbiology, vol. 43, no. 4, pp. 238$243,2001$. 
[20] A. Kuroda, Y. Sugimoto, T. Funahashi, and J. Sekiguchi, "Genetic structure, isolation and characterization of a Bacillus licheniformis cell wall hydrolase," Molecular and General Genetics, vol. 234, no. 1, pp. 129-137, 1992.

[21] S. Torres, A. Pandey, and G. R. Castro, "Organic solvent adaptation of Gram positive bacteria: applications and biotechnological potentials," Biotechnology Advances, vol. 29, no. 4, pp. 442-452, 2011. 

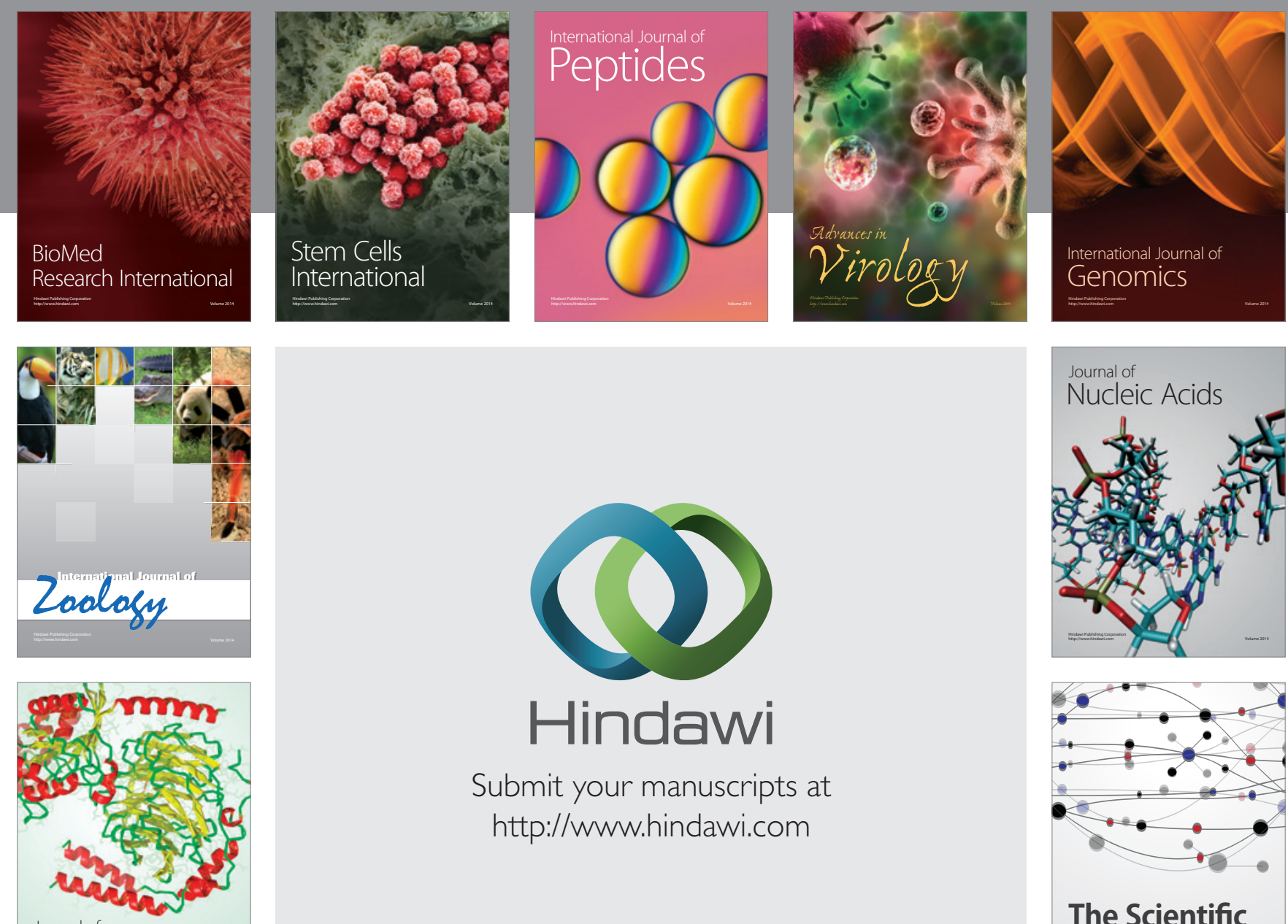

Submit your manuscripts at

http://www.hindawi.com

Journal of
Signal Transduction
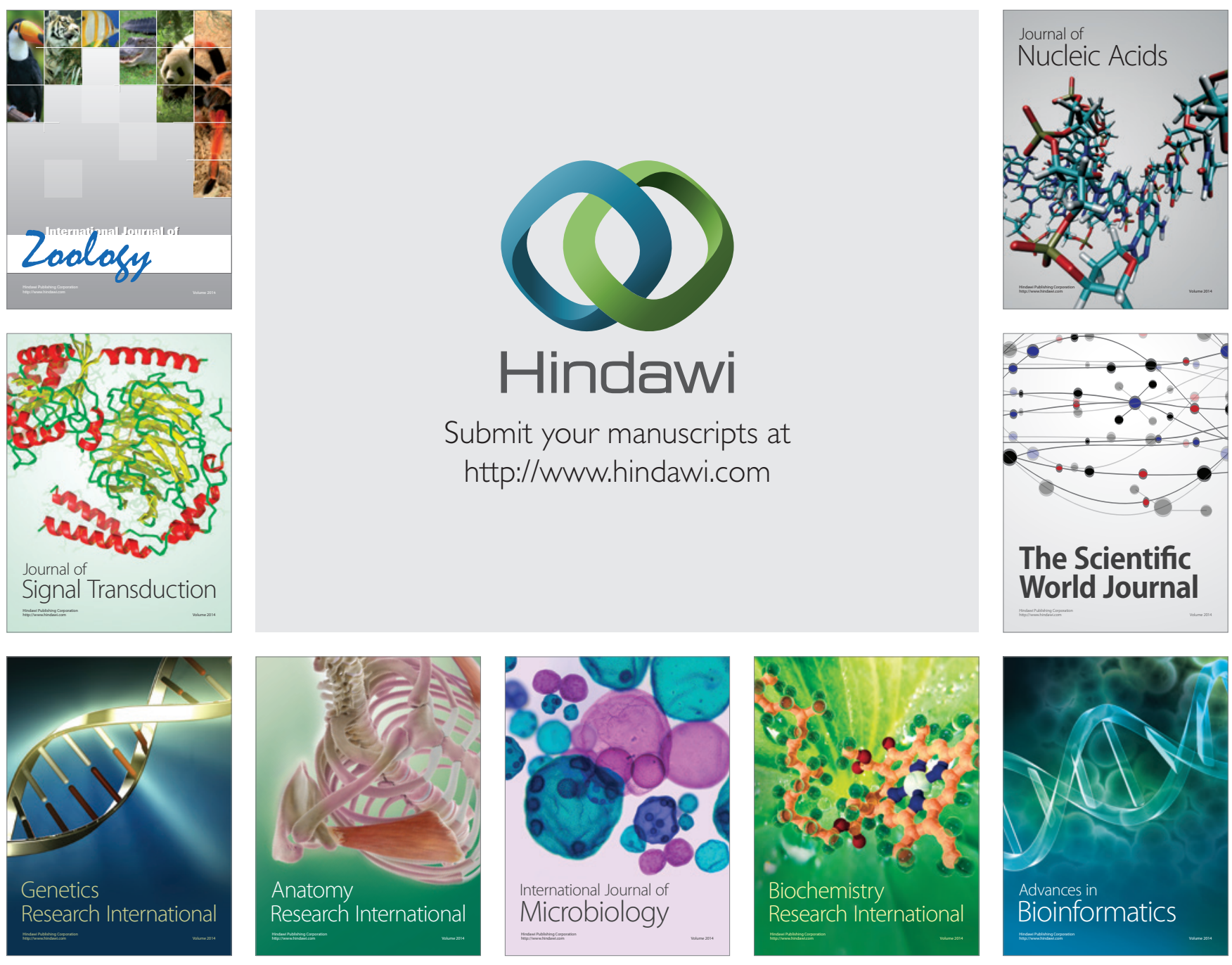

The Scientific World Journal
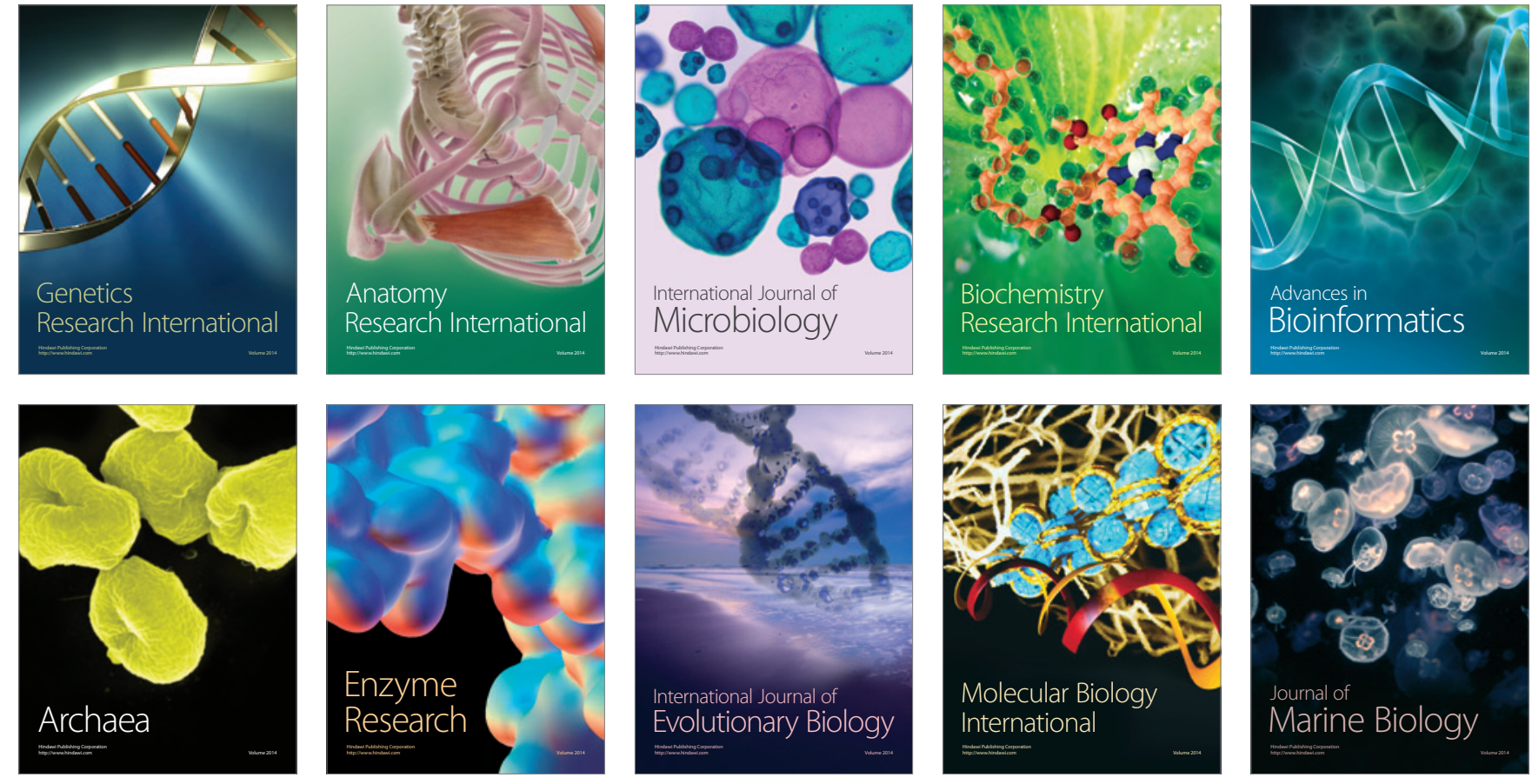